\title{
Endoscopic sinus surgery and musculoskeletal symptoms*
}

\author{
Joanne Rimmer ${ }^{1,2}$, Mohamed Amin33, Wytske J. Fokkens ${ }^{4}$, Valerie J. Lund ${ }^{5,6}$ \\ ' ENT Department, Monash Health, Melbourne, Australia \\ 2 Department of Surgery, Monash University, Melbourne, Australia \\ ENT Department, Mater Misericordiae University Hospital, Dublin, Ireland \\ ${ }^{4}$ Department of Otorhinolaryngology, Academic Medical Centre, Amsterdam, the Netherlands \\ ${ }^{5}$ Professorial Unit, Royal National Throat Nose \& Ear Hospital, London, United Kingdom \\ ${ }^{6}$ Ear Institute, University College London, London, United Kingdom
}

Rhinology 54: 105-110, 2016

DOI:10.4193/Rhino15.217

*Received for publication:

August 19, 2015

Accepted: August 28, 2015

\begin{abstract}
Background: Endoscopic sinus surgery is a common surgical procedure, with low morbidity for patients. Studies have shown that endoscopic and laparoscopic surgeons have a significant risk of developing musculoskeletal symptoms, with potential adverse effects on their careers as well as patient care. We aimed to identify the prevalence of such symptoms, and any associated risk factors relating to surgical technique, in European rhinologists.
\end{abstract}

Methodology: An online survey was distributed to all members of the European Rhinologic Society and data collected for statistical analysis. The relevant literature was reviewed, and ergonomic recommendations made.

Results: There were 250 responses, with nearly $80 \%$ of surgeons experiencing musculoskeletal symptoms. The neck and back were the most common site of symptoms, in approximately $60 \%$ of cases. There were significant correlations between musculoskeletal symptoms and the number of procedures performed each year, operating in a standing position, and operating without a monitor.

Conclusions: There is a high prevalence of musculoskeletal symptoms in endoscopic sinus surgeons, which appears to be particularly related to posture during surgery. Surgeons need to be more aware of the risk factors, and good ergonomic habits should be encouraged to try and reduce the development of such symptoms.

Key words: endoscopic surgical procedures, musculoskeletal system, ergonomics, survey, surgical instruments

\section{Introduction}

Endoscopic sinus surgery (ESS) is accepted as the standard of care for patients with chronic rhinosinusitis refractory to medical treatment, and its extended applications in anterior skull base surgery are ever-increasing. Minimally invasive endoscopic and laparoscopic procedures are widely recognised as offering significant benefits for patients, with reduced morbidity, shorter hospital stays, faster recovery and better cosmetic results. However, they have also been shown to have adverse effects on the physical well being of surgeons ${ }^{(1)}$. The prevalence of musculoskeletal symptoms is significantly higher in endoscopists and minimally invasive surgeons compared to other surgical and medical specialties ${ }^{(2)}$.
A recent survey of British rhinologists found that 59\% experienced pain and/or stiffness as a result of performing endoscopic sinus surgery ${ }^{(3)}$. This is in keeping with the results of an American survey in which $77 \%$ reported musculoskeletal symptoms as a result of performing ESS ${ }^{(4)}$. Both of these studies included relatively small numbers of respondents, so we aimed to determine the prevalence of musculoskeletal symptoms in a larger number of endoscopic sinus surgeons via a pan-European survey.

\section{Methodology}

Study design

A web-based survey of members of the European Rhinologic Society (ERS) was undertaken over a 30 day period. An email containing a hyperlink to the survey website was sent out to 
2235 members of the ERS; members were asked to complete the survey only once. The IP address of the responding computer was logged but no personal information was stored. Entries from duplicate IP addresses were rejected. The survey contained 20 questions (Appendix 1). Members from the UK were excluded as they had already been asked to complete the survey earlier in $2014{ }^{(3)}$, but their responses are compared to those received in the European survey.

After demographic information was obtained, only respondents who perform ESS were asked to complete the remainder of the survey. Details about the number of endoscopic procedures performed each year, and for how many years, were followed by questions relating to their surgical technique (sitting or standing, endoscope or monitor). Information about any musculoskeletal symptoms was collected, including site, severity, duration, diagnosis and any effects the symptoms may have had on work or leisure activities.

\section{Statistical analysis}

Data was coded and analysed using the Statistical Package for the Social Sciences (SPSS) v.22.0 (SPSS Inc., Chicago, IL, USA). Descriptive statistics were used and non-parametric tests were performed.

\section{Results}

There were 250 responses in total, giving a response rate of $11.3 \%$. There was a male preponderance $(78.8 \%)$, as might be expected. The median age of responders was $40-50$ years (35.2\%) (Figure 1). Two-thirds of respondents provided their country of practice (Figure 2), with a good geographical spread of responses from all over Europe, North Africa and the Middle East as well as Japan and Australasia. The highest response rates were from those working in the Netherlands (15.6\%), Denmark (8\%), Sweden and Australia (both 6.8\%).

Ninety-eight per cent of those who responded do perform ESS so were asked to continue; $95.2 \%$ completed the survey. Of these, $32.4 \%$ perform less than 50 endoscopic procedures per year, $44.5 \%$ between 50 and 100 per year, and $23.1 \%$ perform more than 100 endoscopic cases each year (Figure 3). Figure 4 shows the duration of endoscopic practice among respondents. The majority of surgeons (78.6\%) perform ESS standing rather than sitting, and $82.3 \%$ operate from the monitor screen rather than via the endoscope itself. As expected, most are right-handed (94.5\%) and stand on the right side of the table (78.2\%).

Nearly $80 \%$ of surgeons reported some sort of musculoskeletal symptom(s), with only $23.5 \%$ experiencing none. Pain (63.3\%) and stiffness (54.9\%) were the most common symptoms, with paraesthesia in $12.8 \%$. The most frequently affected body areas

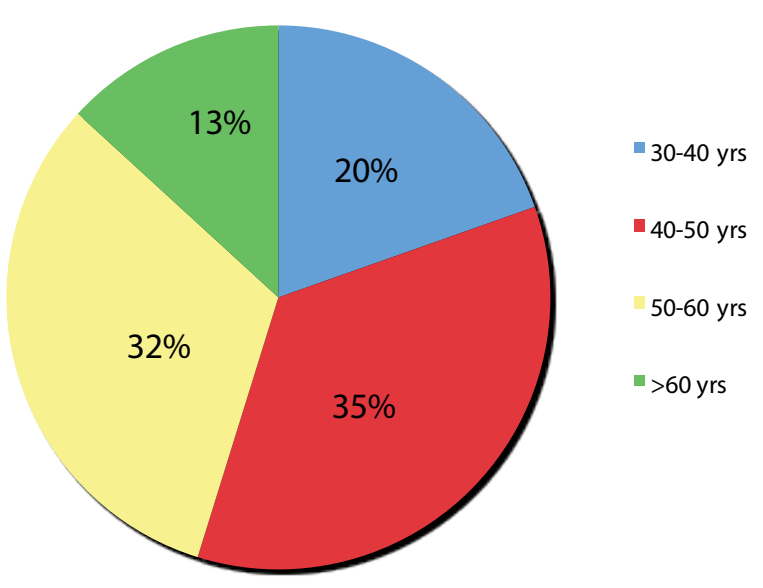

Figure 1. Age distribution of respondents.

were the neck (60.3\%), back (59.8\%) and shoulders (50.8\%), although over $10 \%$ of surgeons also reported symptoms in the fingers (17.9\%), wrists (11.7\%), thumbs (10.6\%) and knees (10.6\%); Figure 4 shows these results in further detail. Whilst symptoms were bilateral in $51.2 \%$, one-third reported that their symptoms were ipsilateral to the endoscope and $16.7 \%$ were contralateral. The majority of surgeons (44.5\%) described their symptoms as ongoing for between one and five years, with $31.8 \%$ for over 10 years. On a pain scale, $53.9 \%$ of respondents rated their pain as mild, $37.5 \%$ moderate and $8.5 \%$ reported severe pain. Just over one quarter of those affected felt that their symptoms affected their ability to enjoy recreational or social activities, but only $7.9 \%$ had ever taken any time off work because of their symptoms. Medical advice and/or treatment had been sought by $26.7 \%$ of surgeons. A specific diagnosis had been made in $19.5 \%$ of respondents, half of which were prolapsed cervical or lumbar vertebral discs. The remaining diagnoses included a traumatic thoracic vertebral fracture, osteoarthritis of the fingers or knees, "tennis elbow" (lateral epicondylitis), and avascular necrosis of the humerus.

Free text comments were invited on suggestions for avoiding the development of musculoskeletal symptoms. The most common of these, in over one third of responses, was to ensure a good posture while operating and take regular rests during procedures. Sitting rather than standing was felt to be helpful by one quarter of those who commented, although the opposite advice was also given. The position of the monitor screen was felt to be important by nearly one-fifth, to avoid twisting or straining. Other suggestions included regular exercise outside the operating theatre, limiting the number of cases per list, and operating more quickly!

There was a statistically significant correlation between musculoskeletal symptoms attributed to the use of the endoscope or body posture during ESS and the number of procedures per- 


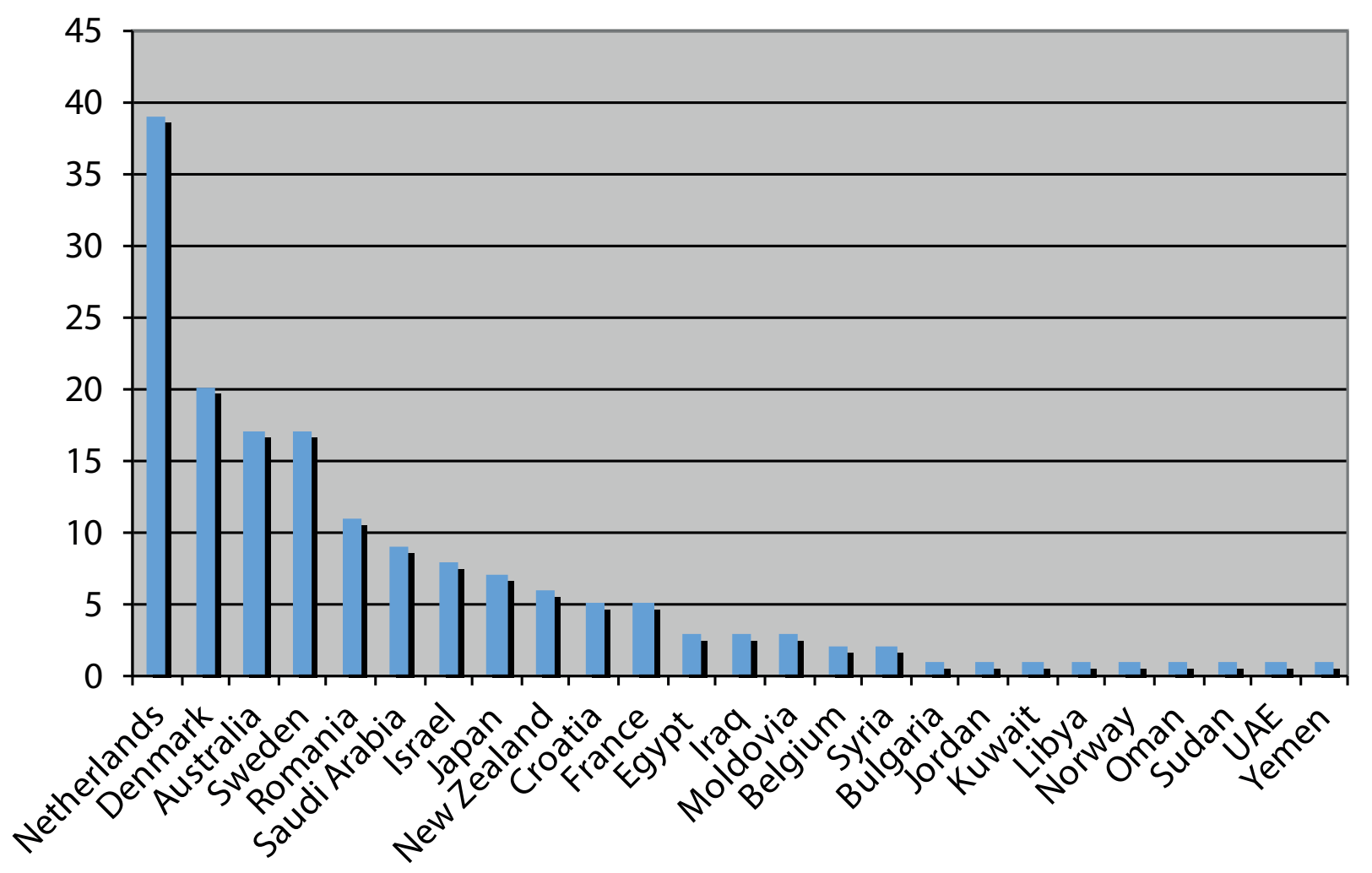

Figure 2. Number of responses by country.

formed per year, with symptoms more likely to occur in those performing a greater number of endoscopic surgeries $(p<0.01)$. Symptoms were significantly more common in those who perform ESS standing up $(p<0.01)$, and in those who operate with a beam-splitter or the endoscope alone $(p<0.01)$ rather than using a monitor. There was also a positive correlation between symptoms and being right-handed $(p<0.01)$ and operating to the right of the table $(p<0.01)$. There was no statistically significant correlation between age group and symptoms.

When these responses are compared to the results of the UK survey, there was a lower percentage response rate for the European survey (11.3\% compared to $22.4 \%$ in the UK survey) ${ }^{(3)}$. The actual number of responses was larger (250 responses compared to 82 in the UK) due to the significantly larger membership of the ERS. The demographics were similar, with a slightly higher percentage of European surgeons (17.7\%) using the endoscope rather than the monitor; in the UK only $7 \%$ do not use the monitor.

A similar percentage of surgeons reported musculoskeletal symptoms in both surveys, with pain and stiffness the most common. The back, neck and shoulders were the most frequent site of symptoms, with over $50 \%$ reporting bilateral symptoms in both surveys. The impact of these symptoms on work or leisure activities was similar across both groups, and a specific diagnosis had been made in the same percentage of surgeons.

Both studies found a positive correlation between symptoms and operating in a standing position. The UK study also reported a positive correlation with increasing age, which was not found in this larger survey.

\section{Discussion}

It has been reported previously that surgeons are at a high risk of developing musculoskeletal symptoms, both as a result of the physical nature of their work and the mental intensity that accompanies it ${ }^{(5)}$. Such symptoms may adversely affect surgeons' long-term health, their productivity and longevity, and could potentially affect patient care. The global mean prevalence of back pain is reported to be approximately $12 \%$, but numerous studies have shown that back pain is experienced by $60-80 \%$ of endoscopic surgeons ${ }^{(6)}$. Endoscopic and laparoscopic surgeons experience similar symptoms ${ }^{(4)}$. "Minimal access surgery-related surgeon morbidity syndromes" have been described, constituting a spectrum of surgery-related injuries, the major factors in which are fatigue, overuse syndrome and imperfect ergonomics (7). Ergonomics is the study of efficiency within the working environment; good ergonomic principles should be applied to make that environment as comfortable and safe as possible for the worker. Two-thirds of ENT surgeons do not feel knowledgeable about ergonomics ${ }^{(4)}$. 


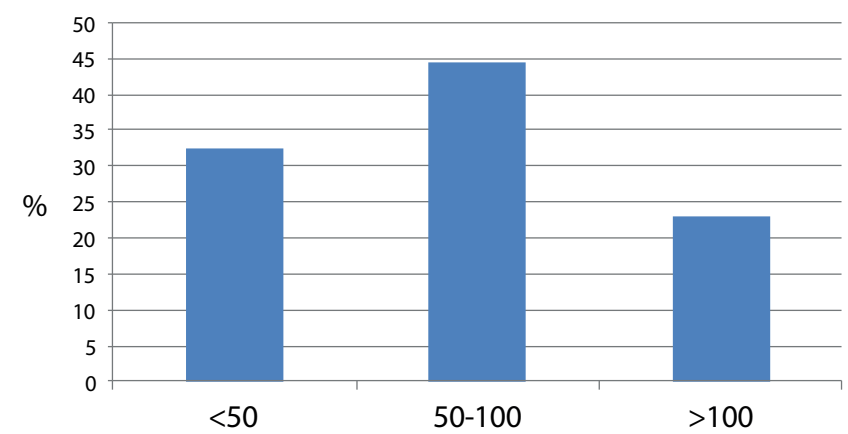

Figure 3. Number of ESS procedures per annum.

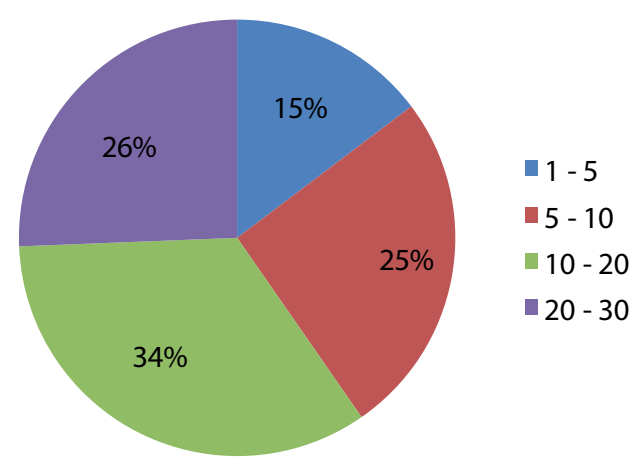

Figure 4. Duration of endoscopic practice in years.
A web-based survey of gynaecologists found symptoms affecting the back in $76 \%$, neck in $73 \%$, shoulder in $66 \%$ and wrist and/or hand in $61 \%{ }^{(5)}$. Thirty-five per cent felt that their surgical performance suffered, and $36 \%$ reported that their hobbies were affected. In similar studies, nearly $80 \%$ of laparoscopic and thoracoscopic surgeons also complained of discomfort in the neck, shoulders and back ${ }^{(8,9)}$. The thoracic surgeons felt that their symptoms were related to a static position, incorrect monitor and table heights and the use of foot pedals ${ }^{(9)}$.

When general surgeons were surveyed, over $80 \%$ reported musculoskeletal symptoms in at least one body area, most commonly the neck (83\%), back (68\%) and shoulder (58\%) ${ }^{(10)}$. Eighty-nine per cent related their symptoms to their posture during surgery. Over $80 \%$ of plastic surgeons reported at least one musculoskeletal injury including muscle strain, neck pain, shoulder arthritis and carpal tunnel syndrome ${ }^{(11)}$. Microscope use was particularly associated with neck and back pain, and this was also noted in a previous survey of UK ENT consultants (12). Neck pain was reported in $78 \%$ and back pain in $63 \%$, both of which were more common in otologists and head and neck surgeons than rhinologists, thought to be due to the static postures and prolonged sitting required for microscope work. Over half of those surveyed attributed their symptoms to work.

An American study found that $73 \%$ of ENT surgeons feel that the ergonomics of ESS cause fatigue, and $77 \%$ reported symptoms as a result of performing ESS ${ }^{(4)}$. Sixty-three per cent described symptoms in the shoulder, $54 \%$ in the hands and $46 \%$ in the neck; $23 \%$ had sought medical advice.

Surveys, whether online or postal, typically have a low response rate. Although we had a large number of respondents, the overall response rate was $11.8 \%$; similar surveys in other surgical specialities report response rates from $7.9 \%$ to $68 \%$, with a median response rate around $20 \%{ }^{(4,5,8-11)}$.

We found a significant correlation between musculoskeletal symptoms and operating standing up, as did the UK study ${ }^{(3)}$. Our results also showed a significant association with being right-handed and operating to the right of the table, but other studies have shown no association with standing or handedness ${ }^{(4)}$. We had larger numbers overall than these studies but the number of left-handed surgeons is small, which may have biased our results.

The link between musculoskeletal symptoms and operating whilst standing up, rather than in a seated position, has long been recognised ${ }^{(13)}$. Prolonged standing is also associated with varicose veins, venous thromboses and intervertebral disc problems ${ }^{(14)}$. Posture is stabilized and sway is reduced by even very light touch such as leaning on the operating table or resting the endoscope on the upper part of the nasal rim ${ }^{(2)}$. Gel floor mats have been shown to produce significant reduction in discomfort in laparoscopic urological procedures ${ }^{(15)}$.

This survey found a significant correlation between operating with a beam-splitter or the endoscope alone, rather than using a monitor, and musculoskeletal symptoms. This is likely to be due the position required to look directly down an endoscope, whereby the trunk and neck are flexed and rotated ${ }^{(14)}$. Proponents of the endoscope/beam splitter suggest that spatial orientation is better than with a monitor, but studies have found no difference in manual precision or complications ${ }^{(16,17)}$. Studies in dentists have shown an association between neck pain and neck flexion and rotation, with symptoms reported in up to $93 \%{ }^{(18-20)}$. This is also thought to be due to the static position adopted for most of their work. Static positions cause increased muscular fatigue with time, and surgeons are more likely to maintain a static posture when concentrating very hard ${ }^{(21)}$. Five key ergonomic factors have been suggested to affect surgeons' posture during minimally invasive surgery: static body posture, foot pedals, monitor position, table height and instrument design ${ }^{(22)}$. Other suggested causes include adopting awkward postures during surgery and repetitive motions. Younger surgeons are perhaps more likely to grip instruments more strongly 


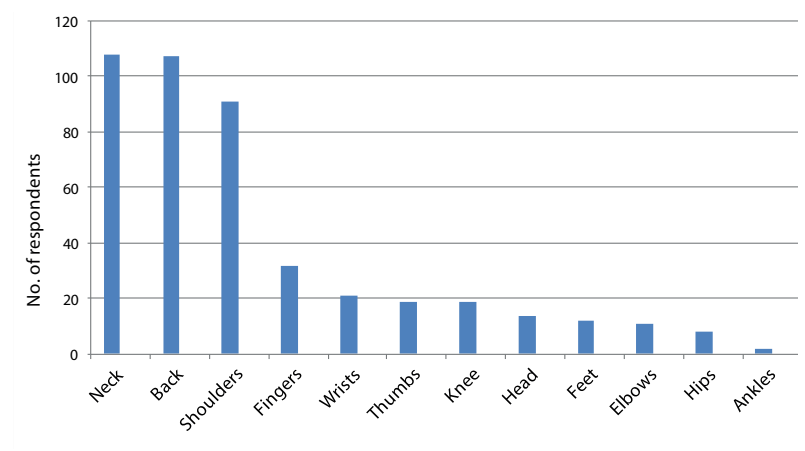

Figure 5. Site of musculoskeletal symptoms.

and assume more contorted positions due to a lack of experience ${ }^{(23)}$. ENT surgeons have been shown to spend over $50 \%$ of their operating time with their heads flexed, rotated or twisted (24). Laparoscopic surgery is associated with a more static posture and significantly higher movements of the shoulder, elbow and wrist ${ }^{(25)}$. Surgeons often hover their foot over pedals, even when sitting, which adds to poor posture and leg discomfort. More ergonomic pedal designs and finger-switch instruments have been suggested as solutions ${ }^{(2)}$.

Monitors should allow for height adjustment so that the surgeon's visual and motor axes can be aligned as much as possible. The monitor should be positioned in front of the surgeon and slightly below eye level at approximately one metre away to minimize eye strain ${ }^{(2,4,14,26)}$. The vertical direction of gaze for minimal eye strain should be 5-35 degrees below the Frankfort plane ${ }^{(26)}$. The optimal table height allows instruments to be at elbow height; if they are held above that then there is increased upper limb muscle work. If the table is too low then trunk flexion increases, and vice versa ${ }^{(2)}$.

Endoscopic instruments require more effort to perform the same manoeuvre compared to an open procedure because instruments are three to nine times less efficient in the transfer of mechanical force from handle to tip ${ }^{(7)}$. Ring-grip instruments require more effort than shank handles and have been reported to cause digital neuropraxia from pressure points ${ }^{(2)}$. Innovative instrument design would improve surgeon comfort and efficiency.

Muscle endurance training has been recommended to reduce surgeon morbidity, and ergonomic principles should be part of surgical training. The Alexander technique, which reportedly improves the positional relationship between the head, neck and spine during activity, has been shown to improve surgeon posture with subsequent reduction in discomfort and fatigue (27). Others have suggested routine conditioning with stretching and exercise during the day ${ }^{(11)}$. Elbow rests have been suggested to reduce activity in neck and shoulder muscles as well as the lower back, and can double endurance time ${ }^{(14)}$. However, the ideal design and height has yet to be found.

\section{Conclusion}

This pan-European survey provides further evidence of the high prevalence of musculoskeletal problems attributed to ESS among rhinologists. Endoscopic skull base procedures are longer and physically more demanding than standard ESS cases, and are becoming more common ${ }^{(28)}$. This will only serve to increase the overall problem. Endoscopic surgeons should be aware of ergonomic factors that may reduce the development of such symptoms, including surgeon posture, monitor position and instrument design.

\section{Authorship contribution}

JR contributed to the design of the survey, collected the results and wrote the paper. MA contributed to the design of the survey, undertook statistical analysis and reviewed the paper. VJL developed the idea, contributed to the design of the survey and undertook senior review of the paper. WF undertook senior review of the paper.

\section{Conflict of interest}

None to declare.

\section{References}

1. Park A, Lee G, Seagull FJ, Meenaghan N Dexter D. Patients benefit while surgeons suffer: an impending epidemic. J Am Coll Surg. 2010;210(3):306-313

2. Ramakrishnan VR, Montero PN. Ergonomic considerations in endoscopic sinus surgery: lessons learned from laparoscopic surgeons. Am J Rhinol Allergy. 2013;27(3):245250.

3. Amin M, Rimmer J, Swift A, White P, Lund VJ. FESS, fingers and other things - you are not alone! Rhinology. 2015;53(2):116-121.

4. Little RM, Deal AM, Zanation AM, McKinney K, Senior BA, Ebert CS, Jr. Occupational hazards of endoscopic surgery. Int Forum Allergy Rhinol. 2012;2(3):212-216.

5. Adams SR, Hacker MR, McKinney JL, Elkadry EA, Rosenblatt PL. Musculoskeletal pain in gynecologic surgeons. J Minim Invasive Gynecol. 2013;20(5):656-660.

6. Hoy D, Bain C, Williams G, March L, Brooks $P$, Blyth $F$, et al. A systematic review of the global prevalence of low back pain. Arthritis Rheum. 2012;64(6):2028-2037.

7. Reyes DA, Tang B, Cuschieri A. Minimal access surgery (MAS)-related surgeon morbidity syndromes. Surg Endosc. 2006;20(1):1-13.

8. Wauben LS, van Veelen MA, Gossot D,
Goossens RH. Application of ergonomic guidelines during minimally invasive surgery: a questionnaire survey of 284 surgeons. Surg Endosc. 2006;20(8):1268-1274.

9. Welcker K, Kesieme EB, Internullo E, Kranenburg van Koppen LJ. Ergonomics in thoracoscopic surgery: results of a survey among thoracic surgeons. Interact Cardiovasc Thorac Surg. 2012;15(2):197-200.

10. Szeto GP, Ho P, Ting AC, Poon JT, Cheng SW, Tsang RC. Work-related musculoskeletal symptoms in surgeons. J Occup Rehabil. 2009;19(2):175-184

11. Capone AC, Parikh PM, Gatti ME, Davidson BJ, Davison SP. Occupational injury in 
plastic surgeons. Plast Reconstr Surg 2010;125(5):1555-1561.

12. Babar-Craig H, Banfield G, Knight J. Prevalence of back and neck pain amongst ENT consultants: national survey. J Laryngo Otol. 2003;117(12):979-982.

13. Xu Y, Bach E, Orhede E. Work environment and low back pain: the influence of occupational activities. Occup Environ Med. 1997:54(10):741-745

14. Ayad T, Peloquin L, Prince F. Ergonomics in endoscopic sinus surgery: systematic review of the literature. J Otolaryngol. 2005;34(5):333-340.

15. Haramis G, Rosales JC, Palacios JM Okhunov Z, Mues AC, Lee D, et al. Prospective randomized evaluation of foot gel pads for operating room staff comfort during laparoscopic renal surgery. Urology. 2010;76(6):1405-1408

16. White PS, Frizelle FA, Hanna GB, Tan LK, Gardiner Q, Cuschieri A. Comparison of direct monocular endoscopic, two- and three-dimensional display systems on surgical task performance in functional endoscopic sinus surgery. Clin Otolaryngol Allied Sci. 1997:22(1):65-67.

17. Tasman AJ, Stammberger H. Videoendoscope versus endoscope for paranasal sinus surgery: influence on stereoacuity. Am J Rhinol. 1998;12(6):389-392.

18. Rundcrantz BL, Johnsson B, Moritz U. Occupational cervico-brachial disorders among dentists. Analysis of ergonomics and locomotor functions. Swed Dent J. 1991;15(3):105-115

19. Finsen L, Christensen $H$, Bakke M. Musculoskeletal disorders among dentists and variation in dental work. Appl Ergon. 1998;29(2):119-125

20. Anton D, Rosecrance J, Merlino L, Cook T. Prevalence of musculoskeletal symptoms and carpal tunnel syndrome among dental hygienists. Am J Ind Med. 2002;42(3):248 257.

21. Berguer R, Rab GT, Abu-Ghaida H, Alarcon A Chung J. A comparison of surgeons' posture during laparoscopic and open surgical procedures. Surg Endosc. 1997;11(2):139-142.

22. van Veelena MA, Jakimowicz JJ Kazemier G. Improved physical ergonomics of laparoscopic surgery. Minim Invasive Ther Allied Technol. 2004;13(3):161-166

23. Hemal AK, Srinivas $M$, Charles AR. Ergonomic problems associated with laparoscopy. J Endourol. 2001;15(5):499-503.

24. Kant IJ, de Jong LC, van Rijssen-Moll M, Borm PJ. A survey of static and dynamic work postures of operating room staff. Int Arch Occup Environ Health. 1992;63(6):423428.

25. Nguyen NT, Ho HS, Smith WD, Philipps C, Lewis C, De Vera RM, et al. An ergonomic evaluation of surgeons' axial skeletal and upper extremity movements during laparoscopic and open surgery. Am J Surg.
2001;182(6):720-724.

26. Jaschinski W, Heuer $\mathrm{H}$, Kylian $\mathrm{H}$. Preferred position of visual displays relative to the eyes: a field study of visual strain and individual differences. Ergonomics. 1998:41(7):1034-1049.

27. Reddy PP, Reddy TP, Roig-Francoli J, Cone $L$, Sivan B, DeFoor WR, et al. The impact of the Alexander technique on improving posture and surgical ergonomics during minimally invasive surgery: pilot study. Urol. 2011;186(4 Suppl):1658-1662.

28. Ensenat J, de Notaris $M$, Sanchez $M$ Fernandez C, Ferrer E, Bernal-Sprekelsen $M$, et al. Endoscopic endonasal surgery for skull base tumours: technique and preliminary results in a consecutive case series report. Rhinology. 2013;51(1):37-46.

\section{Miss Joanne Rimmer \\ Monash ENT Department \\ Moorabbin Hospital \\ 823-865 Centre Road \\ Bentleigh East, VIC 3165 \\ Australia}

Tel: +61-3-9928 8799

Email: rimmer.joanne@gmail.com

Appendix 1: Survey questions.

\begin{tabular}{|c|c|}
\hline Q1 & How old are you? \\
\hline Q2 & Are you male or female? \\
\hline Q3 & What country do you practice in? \\
\hline Q4 & Do you perform endoscopic sinus surgery (ESS)? \\
\hline Q5 & How many ESS procedures do you undertake per year? \\
\hline Q6 & How many years have you been performing ESS? \\
\hline Q7 & In your practice do you perform ESS sitting or standing? \\
\hline Q8 & Are you right or left handed? \\
\hline Q9 & Do you stand on the right side or the left side of the operating table? \\
\hline Q10 & During ESS do you use the endoscope/beam splitter or the monitor screen only to operate? \\
\hline Q11 & $\begin{array}{l}\text { Do you or have you experienced any of these symptoms that you can attribute to the use of endoscopy or your body posture during ESS } \\
\text { procedures: pain stiffness, paraesthesia, or none? }\end{array}$ \\
\hline Q12 & $\begin{array}{l}\text { If you have experienced any of these symptoms that you can attribute to the use of endoscopy or your body posture during ESS, which area } \\
\text { of your body was affected: neck, head, shoulders, elbows, wrists, thumbs, fingers, back, hips, knees, ankles, feet, other (please specify)? }\end{array}$ \\
\hline Q13 & Is the affected area of your body: On the side you hold the endoscope, on the side you hold the surgical instruments or both? \\
\hline Q14 & How long have you suffered with your current symptoms? \\
\hline Q15 & How would you rate the severity of your symptoms on a scale of $0-10$ (10 being the worst pain)? \\
\hline Q16 & Have your symptoms interfered with your hobbies, recreational or social activities? \\
\hline Q17 & Have you required time off work because of the severity of your symptoms? \\
\hline Q18 & Have you sought medical advice, treatment or surgery because of your symptoms? \\
\hline Q19 & Have you had a specific problem diagnosed such as prolapsed disc? \\
\hline Q20 & Have you any comments or advice for new ESS surgeons to avoid/minimize these potential occupational hazards? \\
\hline
\end{tabular}

